\title{
Novel Blockade of Protein Kinase A-Mediated Phosphorylation of AMPA Receptors
}

\author{
Amanda M. Vanhoose, ${ }^{1}$ Julie M. Clements, ${ }^{1}$ and Danny G. Winder ${ }^{1,2}$ \\ ${ }^{1}$ Department of Molecular Physiology and Biophysics, and ${ }^{2}$ Center for Molecular Neuroscience, J. F. Kennedy Center for Research on Human Development, \\ Vanderbilt University School of Medicine, Nashville, Tennessee 37232-0615
}

\begin{abstract}
The phosphorylation state of the glutamate receptor subtype 1 (GluR1) subunit of the AMPA receptor (AMPAR) plays a critical role in synaptic expression of the receptor, channel properties, and synaptic plasticity. Several $\mathrm{G}_{\mathrm{s}}$-coupled receptors that couple to protein kinase A (PKA) readily recruit phosphorylation of GluR1 at S845. Conversely, activation of the ionotropic glutamate NMDA receptor (NMDAR) readily recruits dephosphorylation of the same GluR1 site through $\mathrm{Ca}^{2+}$-mediated recruitment of phosphatase activity. In a physiological setting, receptor activation often overlaps and crosstalk between coactivation of multiple signaling cascades can result in differential regulation of a given substrate. After investigating the effect of coactivation of the NMDAR and the $\mathrm{G}_{\mathrm{s}}$-coupled $\beta$-adrenergic receptor on GluR1 phosphorylation state, we have observed a novel signal that prevents PKA-mediated phosphorylation of GluR1 at serine site 845. This blockade of GluR1 phosphorylation is dependent on cellular depolarization recruited by either NMDAR or AMPAR activation, independent of $\mathrm{Ca}^{2+}$ and independent of calcineurin, protein phosphatase 1 , and/or protein phosphatase $2 \mathrm{~A}$ activity. Thus, in addition to the typical kinase-phosphatase rivalry mediating protein phosphorylation state, we have identified a novel form of phosphoprotein regulation that occurs at GluR1 and may also occur at several other PKA substrates.
\end{abstract}

Key words: AMPA receptor; $\beta$-adrenergic receptor; NMDA receptor; CA1; depolarization; cAMP-dependent protein kinase

\section{Introduction}

A well characterized cellular change that accounts for many forms of NMDA receptor (NMDAR)-dependent synaptic plasticity in area CA1 of hippocampus is modification of the AMPA receptor (AMPAR), the postsynaptic glutamate receptor (GluR) responsible for mediating fast excitatory synaptic transmission. Several forms of AMPAR regulation have been identified, but of particular interest are two phosphorylation sites in the C-terminal tail of the GluR1 subunit that play a critical role in channel localization, biophysical properties, and plasticity (Roche et al., 1996; Derkach et al., 1999; Banke et al., 2000; Chao et al., 2002; Derkach, 2003; Esteban et al., 2003; Lee et al., 2003). The serine 831 site (S831) is phosphorylated by calcium/ calmodulin-dependent kinase II (CaMKII) and protein kinase C (PKC) (Roche et al., 1996; Mammen et al., 1997; Derkach et al., 1999 ) and is readily phosphorylated in response to NMDAR activation (Barria et al., 1997; Lee et al., 2000; Vanhoose and Winder, 2003). A second site of GluR1 regulation occurs at serine 845 (S845), which is phosphorylated by protein kinase A (PKA) (Roche et al., 1996). Several $\mathrm{G}_{\mathrm{s}}$-coupled receptors such, as the $\beta_{1}$-adrenergic receptor $\left(\beta_{1} \mathrm{AR}\right)$ and the $\mathrm{D}_{1}$ dopamine receptor, robustly couple to phosphorylation of GluR1 at S845 (Price et al.,

\footnotetext{
Received June 7, 2005; revised 0ct. 26, 2005; accepted Dec. 12, 2005.

This work was supported by National Institutes of Health Cellular, Biochemical, and Molecular Sciences Training Grant T32 GM08554 (A.M.V.) and National Institute on Drug Abuse Grant DA13699 (D.G.W.).

Correspondence should be addressed to Dr. Danny G. Winder, Department of Molecular Physiology and Biophysics, 23rd and Pierce Avenue South, Room 724B, Robinson Research Building, Vanderbilt University School of Medicine, Nashville, TN 37232-0615. E-mail: danny.winder@vanderbilt.edu.

D0I:10.1523/JNEUROSCI.3572-05.2006

Copyright $\odot 2006$ Society for Neuroscience $\quad$ 0270-6474/06/261138-08\$15.00/0
}

1999; Snyder et al., 2000; Chao et al., 2002; Vanhoose and Winder, 2003). Conversely, NMDAR activation readily recruits dephosphorylation of this site (Kameyama et al., 1998; Lee et al., 1998; Snyder et al., 2003; Vanhoose and Winder, 2003).

From these types of observations, it appears that there may be a typical kinase- and phosphatase-driven tug-of-war that dictates the final phosphorylation state of GluR1 at S845. However, rather than observing a simple additive effect between phosphorylation and dephosphorylation on the S845 site of GluR1 in response to coactivation of the NMDAR and $\beta A R$, we have observed a more unconventional form of regulation such that previous NMDAR activation results in no detection of phosphorylation mediated by the $\beta A R$ (Vanhoose and Winder, 2003). These data imply that, during NMDAR activation, a novel form of regulation at the S845 site of GluR1 occurs, such that a signal is generated that blocks GluR1 phosphorylation by PKA.

Here, we describe experiments in which we have isolated a signal for blockade of GluR1 phosphorylation that is independent of typical phosphatase-mediated dephosphorylation and occurs via depolarization, which is mediated by either NMDAR or AMPAR activation. In addition, blockade occurs downstream of PKA, suggesting alterations at the substrate, GluR1, and/or kinase-substrate interactions. Finally, this depolarizationmediated signal that blocks kinase-mediated regulation of a protein phosphorylation site appears to affect not only GluR1 but several additional PKA substrates.

The unveiling of this means of GluR1 regulation at S845 gives new insight into glutamate-mediated signaling and supports a significant role for timing-dependent $\mathrm{G}_{\mathrm{s}}$-coupled receptor activity in the modulation of NMDAR-dependent long-term potenti- 
ation (LTP). Furthermore, the identification and characterization of a novel form of phospho-protein regulation should broaden consideration for factors that play a role in the phosphorylation state of a protein.

\section{Materials and Methods}

Brain slice preparation and pharmacological manipulations. Hippocampal slices were prepared from 7- to 13-week-old male C57BL/6J mice (The Jackson Laboratory, Bar Harbor, ME). Mice were decapitated under isoflurane anesthesia, hippocampi were dissected from the brain, and 400- $\mu \mathrm{m}$-thick transverse slices were made using a McIlwain chopper. Slices were placed in multi-welled submerged chambers, except for experiments in Figure $4 C$, which were placed in a submerged recording chamber. Slices were bathed in oxygenated artificial CSF (ACSF) (in mM: $124 \mathrm{NaCl}, 4.4 \mathrm{KCl}, 2 \mathrm{CaCl}_{2}, 1.2 \mathrm{MgSO}_{4}, 1 \mathrm{NaH}_{2} \mathrm{PO}_{4}, 10$ glucose, and 26 $\mathrm{NaHCO}_{3}$ ) at $26-28^{\circ} \mathrm{C}$. Modified ACSF was used in the experiments represented in Figure $5 \mathrm{~A}$ in which $124 \mathrm{~mm} N$-methyl-D-glucamine (NMDG) was added to replace $\mathrm{NaCl}$. For all experiments, hippocampal slices were equilibrated in normal ACSF for at least $90 \mathrm{~min}$ and no longer than $170 \mathrm{~min}$ before any pharmacological treatments. Importantly, because of previously observed variability of isoproterenol-initiated phosphorylation of GluR1 across experiments performed on different days, comparisons of pharmacological manipulations were only made within experiments in which slices were maintained and treated in parallel for time-matched and animalmatched controls.

All antagonists and phosphatase inhibitors were applied for $30 \mathrm{~min}$ before receptor activation. For receptor activation, NMDA or AMPA was applied for $6 \mathrm{~min}$, isoproterenol was applied for $3 \mathrm{~min}$, and, when the two agonists were coapplied, NMDA or AMPA was applied initially and isoproterenol was added during the final $3 \mathrm{~min}$. After pharmacological manipulations, slices were transferred directly to a metal surface in dry ice for rapid freezing and microdissection of area CA1. CA1 tissue was then analyzed via Western blotting. NMDA, isoproterenol, D,L-AP-5, AMPA, cyclothiazide, and calyculin A were obtained from Tocris Cookson (Ballwin, MO). Cyclosporin A was obtained from Calbiochem (La Jolla, CA), BAPTA tetrasodium salt and Ro 25-6981 $\left(\left[R-\left(R^{*}, S^{*}\right)\right]-\alpha\right.$-(4-hydroxypheny1)- $\beta$-methyl-4-(phenylmethyl)-1-piperidinepropanol hydrochloride) were obtained from Sigma (St. Louis, MO), and Sp-cAMP-AM was obtained from Biolog (Hayward, CA).

Western blotting. CA1 mini-slices were homogenized in ice-cold homogenization buffer (20 mm TBS, 0.5\% Triton X-100, 2 mm sodium orthovanadate, and $2 \mathrm{~mm} \mathrm{NaF}$ ). Samples were centrifuged at $10,000 \times g$ for $20 \mathrm{~min}$, and the supernatant was analyzed. Protein levels were determined with a BCA protein assay kit, diluted to equal concentrations, mixed with an equal volume of sample buffer (62.5 mm Tris-Cl, $\mathrm{pH} 6.8$, glycerol, $5 \%$ SDS, $0.5 \%$ bromophenol blue, and $5 \% \beta$-mercaptoethanol), and run on a $10 \%$ polyacrylamide resolving gel. Protein was transferred to two Immobilon polyvinylidene difluoride membranes (Pall Corporation, Pensacola, FL) in series. The first blot was probed with specific primary antibodies, whereas the second blot was stained for total protein using colloidal gold (Bio-Rad, Hercules, CA) to grossly verify equal lane loading. In all cases, the first blot was stripped and reprobed with additional primary antibodies. Primary antibodies used include anti-phospho-GluR1, S845 (1:2000), anti-phospho-GluR1, S831 (1:1000), anti-GluR1 (1:2000) (Upstate Biotechnology, Lake Placid NY), and anti-phospho-PKA substrate (1:500) (Cell Signaling Technology, Beverly MA). Phospho-GluR1 protein signals were normalized to GluR1 protein signals, and each condition is represented as a percentage of the average basal samples. To observe variation in basal samples, basal samples run within a single blot were each normalized to the average of all basal samples. Results from repeated experiments were averaged together, and differences were tested by ANOVA followed by a Fisher's PLSD. Analysis of blots probed with the phospho-PKA substrate antibody were not normalized to a total protein level, but protein assays were preformed on homogenates and dilutions were made to obtain equal protein concentrations across samples. Line scans of individual lanes were performed to qualitatively compare total samples, and individual prominent bands were selected for statistical analysis, which was performed as described above with all phospho-protein antibodies.
Electrophysiology. Hippocampal slices were perfused $(2 \mathrm{ml} / \mathrm{min})$ in a submerged chamber at $\sim 28^{\circ} \mathrm{C}$. Field EPSP (fEPSP) recordings were obtained with ACSF-filled glass electrodes (1-3 M $\Omega$ ) positioned in the stratum radiatum of area CA1. A bipolar nichrome stimulating electrode was also placed in stratum radiatum for stimulation of Schaffer collateral afferents ( $0.05 \mathrm{~ms}$ duration). Test stimuli were applied once every $20 \mathrm{~s}$ at a stimulus intensity that elicits an fEPSP slope that was $\sim 40 \%$ of the maximum.

\section{Results}

\section{NMDAR activation regulates the phosphorylation state of} GluR1 at S845 via at least two distinct signaling mechanisms

We and others have shown previously that NMDAR activation regulates GluR1 phosphorylation state at S845 through the activation of serine/threonine phosphatases, protein phosphatase 1 (PP1) and/or PP2A and PP2B, which recruit robust dephosphorylation (Kameyama et al., 1998; Lee et al., 1998; Vanhoose and Winder, 2003). In addition, we have reported previously a potential second means of NMDAR-mediated regulation of GluR1 phosphorylation state that is manifest when a $\mathrm{G}_{\mathrm{s}}$-coupled receptor that recruits GluR1 phosphorylation at S845 is coactivated with the NMDAR. Preapplication of NMDA for 3 min followed by the addition of isoproterenol, a $\beta A R$ agonist, for 3 min does not result in an additive effect between dephosphorylation and phosphorylation of GluR1, as might have been predicted. Rather, a dominant NMDAR-mediated dephosphorylation with no detectable phosphorylation of GluR1 at S845 is observed (Vanhoose and Winder, 2003), suggesting that NMDAR activation recruits a signal that mediates blockade of $\beta \mathrm{AR}$-mediated regulation of GluR1.

Consistent with our previous report (Vanhoose and Winder, 2003), we show here that application of $50 \mu \mathrm{M}$ NMDA for $6 \mathrm{~min}$ to acutely prepared hippocampal slices results in immediate dephosphorylation of GluR1 at S845 in area CA1 (Fig. 1). This paradigm of NMDA treatment reversibly decreases synaptic transmission, suggesting no gross decrease in slice viability (data not shown). Within the same paradigm, activation of the $\mathrm{G}_{\mathrm{s}}$ coupled $\beta \mathrm{AR}$ with $1 \mu \mathrm{M}$ isoproterenol for $3 \mathrm{~min}$ elicits a rise in phosphorylation of GluR1 at S845 that is completely blocked if NMDA is applied $3 \mathrm{~min}$ previously (Fig. 1). Also shown here, both dephosphorylation and blockade of phosphorylation are inhibited by pretreatment with the NMDAR antagonist D,L-AP-5 (100 $\mu \mathrm{M}, 30 \mathrm{~min}$ pretreatment) (Fig. 1), indicating that both forms of GluR1 regulation occur specifically through NMDAR activation.

These two forms of GluR1 regulation, (1) dephosphorylation and (2) blockade of phosphorylation, observed during NMDAR activation are readily distinguished from each other. First, the NMDA concentration-response curves for each of these two signals are distinct. GluR1 phosphorylation by isoproterenol is blocked with $10 \mu \mathrm{M}$ NMDA, whereas, within the same experiment, dephosphorylation of GluR1 is only observed at $20 \mu \mathrm{M}$ and higher concentrations of NMDA (Fig. $2 \mathrm{~A}$ ). Both of these concentration-response curves were generated by applying varying concentrations of NMDA for 6 min to hippocampal slices with or without coapplication of isoproterenol $(1 \mu \mathrm{M})$ during the final 3 min. The differences in the potency of NMDA at eliciting these two effects suggests the possibility that they are mediated by two distinct signaling cascades that are differentially recruited by the NMDAR, with blockade of GluR1 phosphorylation occurring with greater potency than NMDAR-induced dephosphorylation.

Additional evidence for two distinct NMDAR-mediated signals is observed during pretreatment with inhibitors of PP1 and PP2A ( $1 \mu \mathrm{M}$ calyculin A, 30 min pretreatment) and calcineurin 


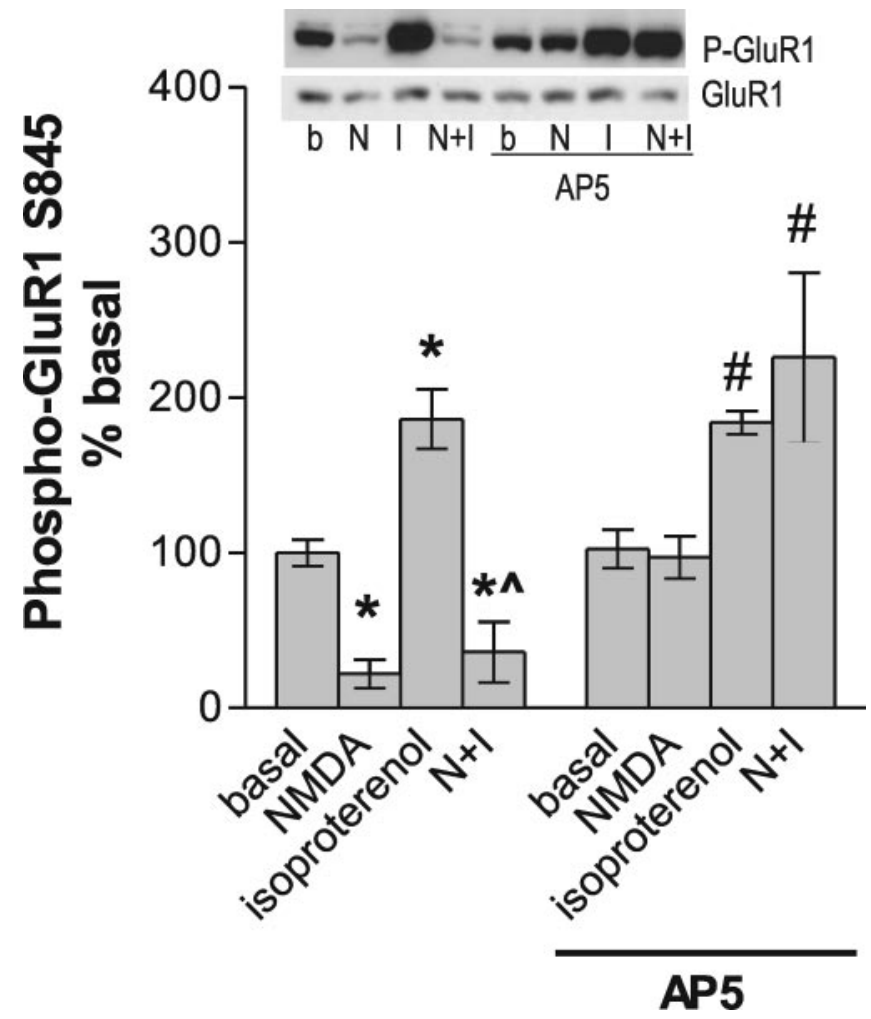

Figure 1. NMDAR activation blocks $\beta_{1} A R$-mediated phosphorylation of GluR1 at $\$ 845$. Hippocampal slices were treated with $50 \mu \mathrm{m}$ NMDA for $6 \mathrm{~min}(\mathrm{~N}), 1 \mu \mathrm{m}$ isoproterenol for $3 \mathrm{~min}$ (I), $50 \mu \mathrm{m}$ NMDA for 6 min with $1 \mu \mathrm{m}$ isoproterenol during the final $3 \mathrm{~min}(\mathrm{~N}+\mathrm{I})$, and each of the same conditions in the presence of $100 \mu \mathrm{M}$ D,L-AP-5, applied 30 min previously. Differences in GluR1 phosphorylation (P-GluR1) at S845 normalized to GluR1 were obtained via Western blot of area CA1 tissue and are plotted as percentage differences from basal samples (b). Each treatment condition is displayed from a representative blot as an inset. ${ }^{*} p<0.05$ versus basal; ${ }^{\wedge} p<0.05$ versus isoproterenol; ${ }^{\sharp} p<0.05$ versus $\mathrm{AP}-5 ; n=4$.

(also called PP2B; $1 \mu \mathrm{M}$ cyclosporin A, 30 min pretreatment). In the presence of these inhibitors, there is no detectable effect on the basal phosphorylation state of GluR1 at S845, but NMDARmediated dephosphorylation is inhibited (Fig. 2B) (Vanhoose and Winder, 2003). Conversely, NMDAR-mediated blockade of GluR1 phosphorylation by isoproterenol remains intact (Fig. $2 B$ ). These data thus identify two distinct signaling mechanisms by which NMDAR activation can negatively regulate GluR1 phosphorylation state: one mechanism that is dependent on PP1, PP2A, and/or calcineurin activity and mediates dephosphorylation, and a second mechanism that is independent of these phosphatases and mediates blockade of phosphorylation.

\section{NMDAR-induced regulation of GluR1 phosphorylation at S845 is not blocked by NR2B subunit-specific antagonists} The diversity of NR2 subunits of the NMDAR has been shown to impart NMDARs with distinct molecular and kinetic properties, and thus these distinct NMDAR-mediated signals that differentially regulate GluR1 phosphorylation could be mediated via distinct NMDAR subtypes. Evidence suggests that, in the adult hippocampus, NR2B subunit-containing NMDARs are enriched in the extrasynaptic population, whereas NR2A-containing receptors are enriched in the synaptic population (Cull-Candy and Leszkiewicz, 2004). To begin to test the specific involvement of NMDAR subpopulations in NMDAR-mediated blockade of GluR1 phosphorylation or dephosphorylation, we used the noncompetitive, NR2B-specific antagonists ifenprodil $(10 \mu \mathrm{M}, 15$ min pretreatment) (Fig. 3) and Ro 25-6981 (data not shown). Neither NMDAR-mediated dephosphorylation nor blockade of phosphorylation of GluR1 at S845 are mediated solely via the NR2B subunit-containing NMDARs, because both signals remain intact in the presence of these antagonists. Although these data indicate that NR1/NR2B heteromers are not solely responsible for regulation of GluR1 phosphorylation state, these data do not rule out the possibility that GluR1 phosphorylation could be regulated specifically by other types of NMDARs, including NR1/ NR2A/NR2B heteromers, because the pharmacological agents used here are much less effective on heterotrimeric NMDARs (Hatton and Paoletti, 2005).

NMDAR-mediated blockade of GluR1 phosphorylation at S845 is observed in the absence of extracellular $\mathrm{Ca}^{2+}$

To characterize the NMDAR-mediated signal responsible for blockade of GluR1 phosphorylation, we first tested dependency on extracellular $\mathrm{Ca}^{2+}$, because many NMDAR-mediated signals occur via $\mathrm{Ca}^{2+}$ influx. Extracellular $\mathrm{Ca}^{2+}$, and thus $\mathrm{Ca}^{2+}$-carried current through the NMDAR, was removed by pretreatment of hippocampal slices with $3 \mathrm{~mm}$ BAPTA for $10 \mathrm{~min}$. This treatment successfully blocked several known $\mathrm{Ca}^{2+}$-dependent NMDARmediated signals, including dephosphorylation of GluR1 at S845 (Fig. 4A), phosphorylation of the CaMKII/PKC site S831 on GluR1 (Fig. $4 B$ ), and extracellular signal-regulated kinase phosphorylation (data not shown). In addition, as measured by field recordings in area CA1, this paradigm of BAPTA treatment completely blocks synaptic transmission, which is dependent on presynaptic $\mathrm{Ca}^{2+}$ channel activity, but does not alter fiber volley responses, which are dependent on $\mathrm{Na}^{+}$channel activity (Fig. $4 C)$. Surprisingly, in the presence of BAPTA, NMDA pretreatment continues to recruit a signal for blockade of $\beta A R$-mediated phosphorylation of GluR1 at S845 (Fig. 4A), suggesting that the blockade signal does not require $\mathrm{Ca}^{2+}$ influx during NMDAR activation. Note that blockade of GluR1 phosphorylation is not complete in the presence of BAPTA, because there is some detectable isoproterenol-mediated GluR1 phosphorylation (Fig. 4A); however, this may be attributable to the removal of $\mathrm{Ca}^{2+}$ as a charge carrier that contributes to NMDAR-mediated depolarization rather than $\mathrm{Ca}^{2+}$ as a second messenger (see next section) (Fig. 5).

\section{Depolarization mediates blockade of GluR1 phosphorylation at $\mathrm{S} 845$}

The persistence of the blockade of GluR1 phosphorylation in the presence of BAPTA suggests that NMDAR-induced recruitment of this process involves an unusual source, likely either direct ligand-induced conformational change of the NMDAR or depolarization itself. To more directly test the role of depolarization in NMDAR-mediated blockade of GluR1 phosphorylation, we replaced the major charge carrier through the NMDAR $\mathrm{Na}^{+}$with NMDG, an NMDAR-impermeable monovalent cation. During transfer of hippocampal slices to modified ACSF containing no $\mathrm{Na}^{+}$but rather NMDG and BAPTA, NMDAR activation no longer blocks isoproterenol-mediated GluR1 phosphorylation (Fig. 5A). Thus, depolarization recruited during NMDAR activation is required for blockade of GluR1 phosphorylation. We therefore hypothesized that other means of membrane depolarization may also recruit a blockade of GluR1 phosphorylation.

Under most physiological circumstances, the NMDAR is activated only after depolarization is generated via AMPAR activity. If the blockade in GluR1 phosphorylation is induced simply by membrane depolarization, then one would predict that this 


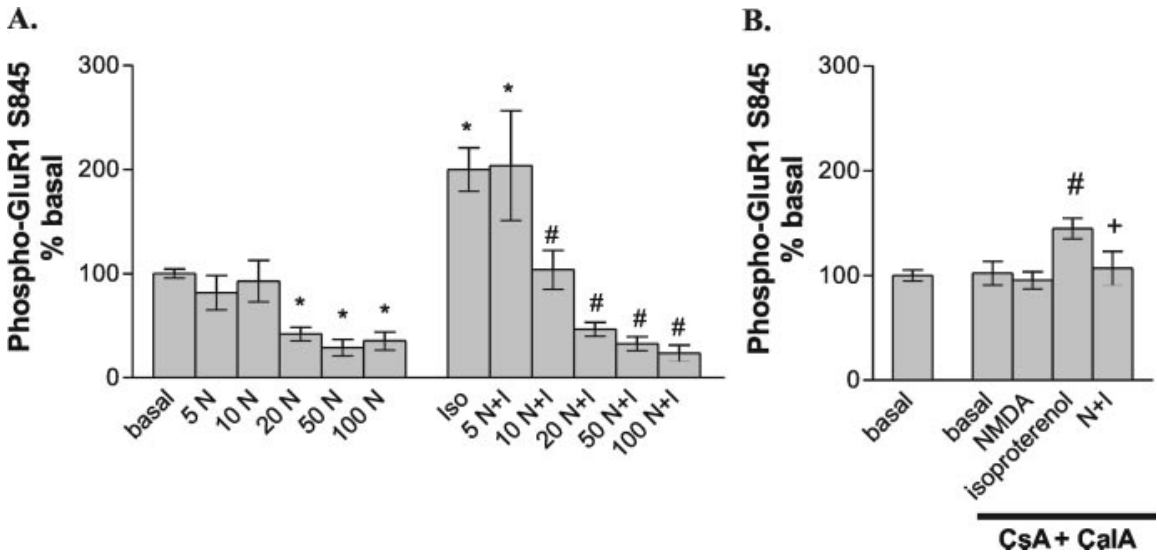

Figure 2. NMDAR activation regulates the phosphorylation state of GluR1 at $\$ 845$ via at least two distinct signaling mechanisms. $A$, An NMDA dose-response curve was generated with 5, 10, 20, 50, and $100 \mu \mathrm{M}$ NMDA applied to hippocampal slices for $6 \mathrm{~min}$ alone ( $\mathrm{N}$ ) or in conjunction with $1 \mu \mathrm{m}$ isoproterenol (Iso) during the final $3 \mathrm{~min}(\mathrm{~N}+\mathrm{I}$ ). Differences in GluR1 phosphorylation at $\$ 845$ normalized to GluR1 were obtained via Western blot of area CA1 tissue and are plotted as percentage differences from basal samples (b). NMDA at $20 \mu \mathrm{m}$ is the threshold dose that induces significant dephosphorylation of $G$ luR 1 at $\$ 845$, whereas 10 $\mu \mathrm{MNMDA}$ is the threshold dose that significantly blocks isoproterenol-mediated GluR1 phosphorylation at $5845 .{ }^{*} p<0.05$ versus basal; ${ }^{\#} p<0.05$ versus isoproterenol; $n=4-14$. B. Hippocampal slices were pretreated with $1 \mu \mathrm{m}$ cyclosporine $A$ and $1 \mu \mathrm{m}$ calyculin A (CsA + CalA) for 30 min before application of $50 \mu \mathrm{m} \mathrm{NMDA} \mathrm{for} 6 \mathrm{~min}$ and/or $1 \mu \mathrm{m}$ isoproterenol for $3 \mathrm{~min}$. CsA + CalA basal samples were not significantly different from vehicle-treated basal samples. In the presence of CsA+CaIA, NMDA-mediated dephosphorylation was undetected, but significant isoproterenol-mediated phosphorylation and NMDA-mediated block of isoproterenol-mediated phosphorylation of GluR1 were detected. ${ }^{\#} p<0.05$ versus CsA + CalA basal; ${ }^{+} p<0.05$ versus CsA + CalA Iso; $n=6$.

blockade is most readily recruited in vivo by direct AMPAR activation. Thus, we applied AMPA (50 $\mu \mathrm{M}$ AMPA, 6 min) to hippocampal slices in the presence of cyclothiazide $(100 \mu \mathrm{M}, 6 \mathrm{~min}$ coapplication with AMPA), a pharmacological agent that prevents AMPAR desensitization. In addition, slices were pretreated with BAPTA ( $3 \mathrm{~mm}, 10 \mathrm{~min}$ pretreatment) to remove $\mathrm{Ca}^{2+}$ mediated signals from subsequently activated voltage-gated $\mathrm{Ca}^{2+}$ channels shown previously to decrease phosphorylation of GluR1 at S845 (Snyder et al., 2003). Under these conditions, AMPAR activation completely blocks detectable GluR1 phosphorylation by isoproterenol, and furthermore, this blockade is not affected by AP-5, an NMDAR antagonist (Fig. 5B). These data are consistent with the conclusion that depolarization alone is sufficient to block GluR1 phosphorylation at S845. Furthermore, because ligand-mediated conformational changes should be receptor specific and independent of ion flux, these data also argue against the possibility that ligand-induced conformational change is responsible for NMDAR-mediated blockade of GluR1 phosphorylation.

\section{Blockade of GluR1 phosphorylation occurs downstream of PKA activation}

We have shown that treatment of hippocampal slices with isoproterenol elicits an increase in phosphorylation of GluR1 at S845 in area CA1, and this receptor-substrate coupling is blocked by depolarization. This blockade could occur via a signal that alters $\beta$ AR signaling or could occur at a level downstream of the receptor, i.e., adenylyl cyclase activity, PKA activity, or GluR1 availability.

To address these possibilities, we incubated hippocampal slices with a cell-permeable direct activator of PKA, Sp-cAMPAM. The kinetics of GluR1 phosphorylation by Sp-cAMP-AM are much slower than that by isoproterenol, because $\mathrm{Sp}$ cAMP-AM must permeate the cell membrane and accumulate intracellularly to directly activate PKA. A time course was generated to determine the minimal time required for Sp-cAMP-AM treatment to elicit a significant rise in GluR1 phosphorylation, which was determined to be $10-15 \mathrm{~min}$, with no detectable effect at 6 min of treatment (data not shown). Because of the potential excitotoxic effects of a 10-15 min NMDA treatment, we added Sp-cAMP-AM for $12 \mathrm{~min}$ and applied NMDA during the last $6 \mathrm{~min}$ of Sp-cAMP-AM treatment, because this is the time period in which Sp-cAMP-AM elicits detectable GluR1 phosphorylation. In addition, this experiment was performed in the presence of calyculin A and cyclosporin A $(1 \mu \mathrm{M}, 30 \mathrm{~min}$ pretreatment) to isolate NMDAR-mediated blockade of GluR1 phosphorylation from NMDAR-mediated dephosphorylation (Fig. 2). The result of NMDAR activation during direct PKA activation by $\mathrm{Sp}$ cAMP-AM is a blockade of GluR1 phosphorylation, because a significant difference in GluR1 phosphorylation is induced by NMDA only in the presence of SPcAMP-AM (Fig. 6). Thus, blockade of GluR1 phosphorylation likely occurs downstream of direct PKA activation, for example, via an alteration in the coupling of PKA to GluR1.

\section{Depolarization blocks phosphorylation of several PKA substrates}

To investigate the specificity or generality of depolarizationdependent blockade of phosphorylation, we used an antibody that detects the phosphorylated state of phospho-proteins that are substrates for PKA to monitor phosphorylation of many proteins simultaneously as described previously (Zhang et al., 2001; Maas et al., 2005). CA1 tissue that has been treated with NMDA before isoproterenol (as in the above-described experiments represented in Fig. 1) was analyzed by immunoblot with a phosphoPKA substrate antibody. As expected, isoproterenol does increase the phosphorylation state of many detectable proteins. Furthermore, NMDA pretreatment blocks several, but not all, isoproterenol-mediated rises in phosphorylation. In Figure $7 C$, a representative blot is displayed with aligned density lines scans for each condition. To illustrate that certain bands appear to be under the influence of an NMDAR-mediated blockade of phosphorylation, we chose a single band at $\sim 80 \mathrm{kDa}$ for quantification that is elevated by treatment with isoproterenol but blocked if pretreated with NMDA (Fig. 7A) $(n=5)$. Also, the NMDARmediated blockade of phosphorylation is blocked by D,L-APV treatment (data not shown). Because not all protein bands exhibit sensitivity to NMDAR-mediated blockade, we also quantified a band below $\sim 80 \mathrm{kDa}$ as one example of this (Fig. $7 B)(n=$ 5 ). These data indicate that depolarization-mediated blockade of phosphorylation may occur at multiple PKA substrates, and thus, during periods of depolarizing activity, PKA-mediated signaling to multiple substrates may be limited.

\section{Discussion}

We have identified a novel mechanism by which the phosphorylation state of GluR1 at S845 is regulated in an acute hippocampal slice preparation. It has been established previously that $\mathrm{G}_{\mathrm{s}}$ coupled receptors, which couple to the PKA cascade, can recruit 


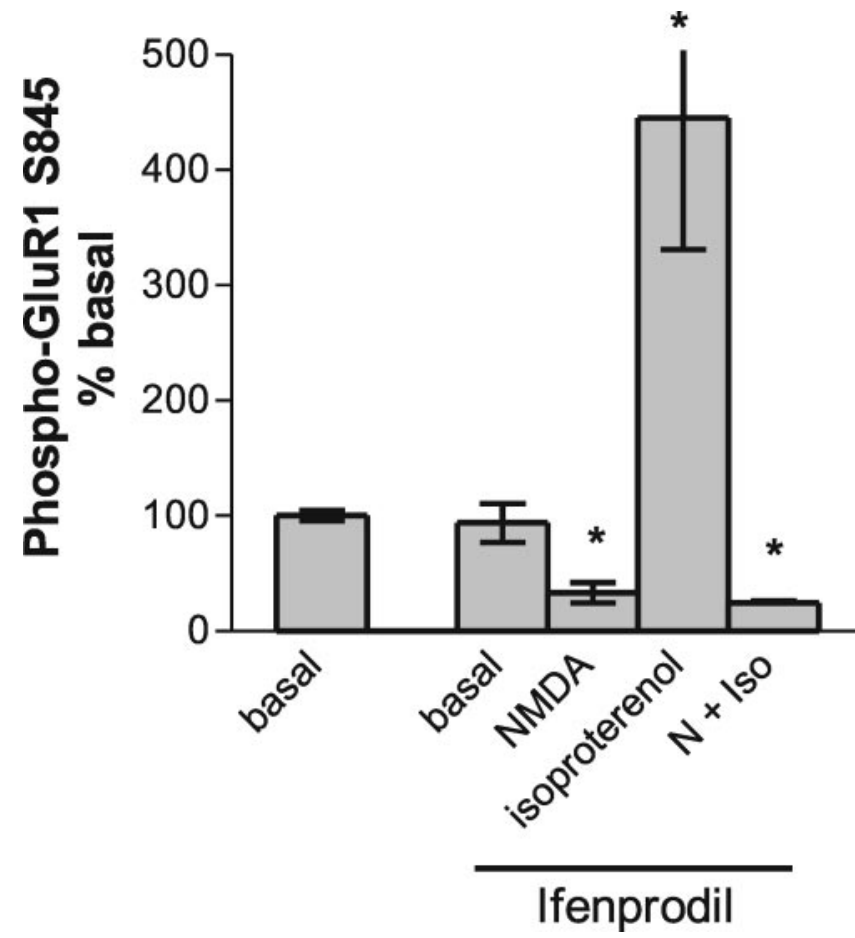

Figure 3. NMDAR-induced regulation of $\$ 845$ phosphorylation is not blocked by NR2B subunit-specific antagonists. Hippocampal slices were pretreated with $10 \mu \mathrm{m}$ ifenprodil for 15 min before application of $50 \mu \mathrm{m}$ NMDA (N) for 6 min and/or $1 \mu \mathrm{m}$ isoproterenol (Iso) for $3 \mathrm{~min}$. Ifenprodil-treated basal samples were not significantly different from vehicle-treated basal samples. In the presence of ifenprodil, NMDA-mediated dephosphorylation and blockade of phosphorylation were both detected. ${ }^{*} p<0.05$ versus basal; $n=4-8$.

GluR1 phosphorylation at S845, and phosphatase activity recruited by the NMDAR can mediate dephosphorylation of the same site. With the data presented here, we have described another layer of phospho-protein regulation that is distinct from the typical kinase-phosphatase rivalry that is commonly known to regulate phospho-proteins. Specifically, depolarization via $\mathrm{Na}^{+}$influx through glutamate receptors results in a blockade of GluR1 phosphorylation by PKA. In addition, this novel mechanism likely serves as a form of regulation for multiple PKA substrates.

The blockade of GluR1 phosphorylation at S845 occurs independent of $\mathrm{Ca}^{2+}$ influx, because BAPTA does not prevent blockade of GluR1 phosphorylation. We cannot completely rule out the possibility of a residual $\mathrm{Ca}^{2+}$ current, but we have demonstrated that, under our experimental conditions, BAPTA treatment causes loss, below detectable levels, of several $\mathrm{Ca}^{2+}$. sensitive effects (Fig. 4). Also, it is important to note that the persistence of the blockade signal in the presence of extracellular BAPTA indicates that blockade is not produced by an intercellular circuit signal, because, under these conditions, evoked transmitter release is blocked. Therefore, blockade of GluR1 phosphorylation at S845 is likely a local, synaptic/dendritic event.

Although independent of extracellular $\mathrm{Ca}^{2+}$, blockade of GluR1 phosphorylation at S845 is dependent on membrane depolarization. Depolarization can alter the activity of voltagesensitive proteins by altering protein conformation and/or protein-protein interactions. Previous reports have shown that G-protein coupled receptor (GPCR) signaling can be regulated in a voltage-sensitive manner (Ben-Chaim et al., 2003), but our data suggest that blockade of $\beta$ AR-mediated GluR1 phosphorylation occurs downstream of both GPCR and PKA activity. Consistent
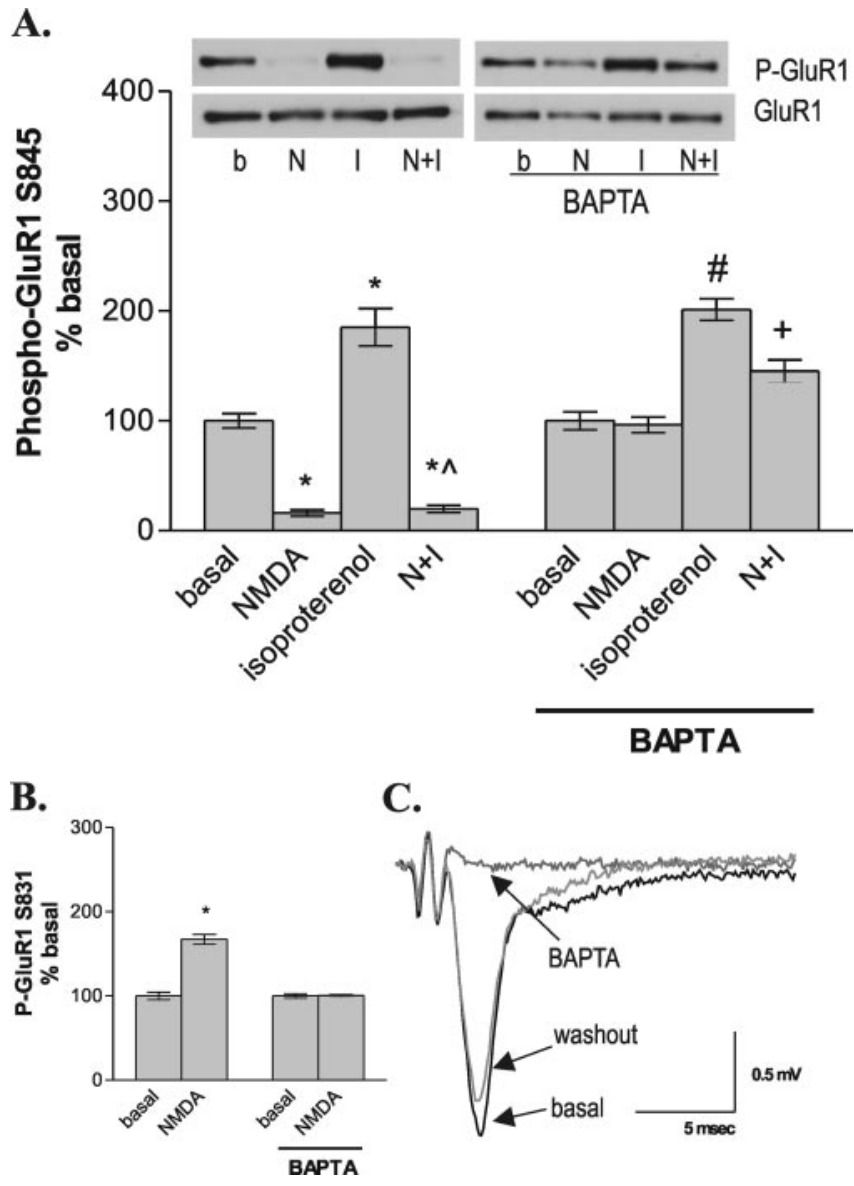

Figure 4. NMDAR-mediated blockade of GluR1 phosphorylation at $\$ 845$ is observed in the presence of BAPTA. A, NMDA (50 $\mu \mathrm{M}, 6 \mathrm{~min}$ ) (N) and/or isoproterenol (1 $\mu \mathrm{M}, 3 \mathrm{~min}$ ) (I) was applied to hippocampal slices in the presence or absence of $3 \mathrm{~mm}$ BAPTA, which was added 10 min before agonist application. Differences in GluR1 phosphorylation (P-GluR1) at 5845 normalized to GluR1 were obtained via Western blot of area CA1 tissue and are plotted as percentage differences from basal samples in the absence of BAPTA. Each treatment condition is displayed from a representative blot as an inset. $\boldsymbol{B}$, The same tissue analyzed in $\boldsymbol{A}$ was also analyzed for phosphorylated GluR1 at S831, normalized to total GluR1 and plotted as a percentage of basal in the absence of BAPTA. C, Representative traces of averaged fEPSPs recorded in area CA1 before BAPTA treatment (basal), during $3 \mathrm{~mm}$ BAPTA treatment (BAPTA), and at $15 \mathrm{~min}$ of washout of BAPTA (washout). ${ }^{*} p<0.5$ versus basal; ${ }^{\wedge} p<0.05$ versus isoproterenol; ${ }^{\#} p<$ 0.05 versus BAPTA basal; ${ }^{+} p<0.05$ versus BAPTA isoproterenol; $n=6$.

with the theory that membrane depolarization promotes blockade of GluR1 phosphorylation at a level downstream of PKA, PKA-mediated GluR1 phosphorylation was not observed in an in vitro postsynaptic density membrane preparation, which maintains many protein-protein interactions but likely does not maintain a polarized membrane state (Vinade and Dosemeci, 2000). However, there is currently no evidence for a specific candidate voltage-sensitive domain or associated protein that could mediate blockade of GluR1 phosphorylation. An alternative to a voltage-mediated effect is that $\mathrm{Na}^{+}$influx during NMDAR or AMPAR activation could serve as a second messenger. Indeed, previous reports have indicated that $\mathrm{Na}^{+}$likely plays a role in signal transduction within neurons (Linden et al., 1993; Yu and Salter, 1998).

The downstream target of $\mathrm{Na}^{+}$influx/depolarization that produces a blockade of PKA-mediated phosphorylation of GluR1 has yet to be determined. Several reports now suggest that GluR1 phosphorylation by PKA within an intact cell preparation is critically dependent on a protein complex that associates PKA with 
A.

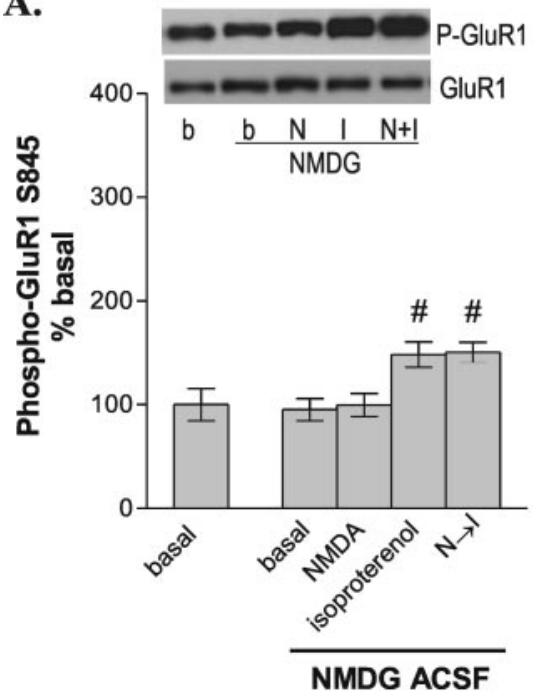

B.

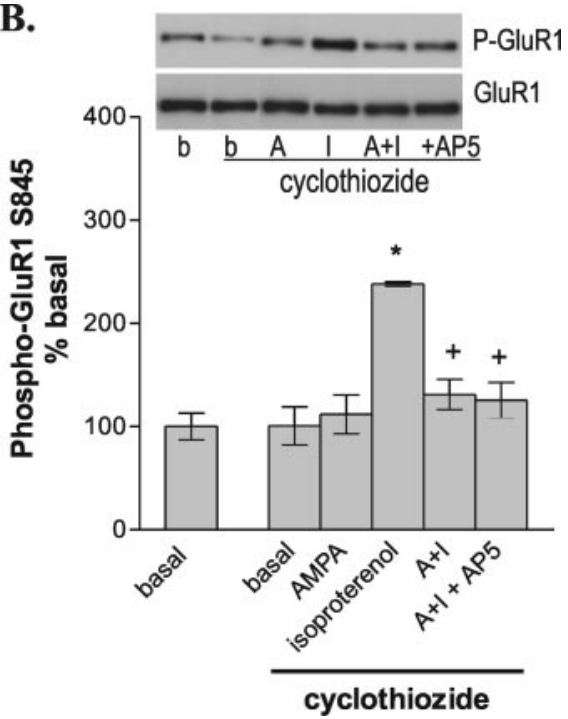

Figure 5. Depolarization mediates blockade of GluR1 phosphorylation (P-GluR1) at S845. A, After postdissection recovery in normal ACSF, hippocampal slices were transferred to $\mathrm{Na}^{+}$-free ACSF that was substituted with NMDG and contained $3 \mathrm{~mm}$ BAPTA. Slices were incubated in the BAPTA/NMDG ACSF for $10 \mathrm{~min}$ before application of NMDA ( $50 \mu \mathrm{m}, 6 \mathrm{~min}$ ) (N) and/or isoproterenol (1 $\mu \mathrm{m}, 3 \mathrm{~min}$ ) (I). ${ }^{*} p<0.05$ versus BAPTA/NMDG basal; $n=3-4$. b, Basal. $\boldsymbol{B}$, Hippocampal slices were pretreated with $3 \mathrm{~mm}$ BAPTA for $10 \mathrm{~min}$ before receiving cyclothiazide ( $100 \mu \mathrm{M}, 6 \mathrm{~min}$ ), AMPA ( $50 \mu \mathrm{M}, 6 \mathrm{~min}$ ), and/or isoproterenol ( $1 \mu \mathrm{M}, 3 \mathrm{~min}$ ). Some slices received a 30 min pretreatment of $100 \mu \mathrm{M}, \mathrm{L}-\mathrm{AP}-5$ before AMPA and isoproterenol treatment (A+I+AP5). Each treatment condition is displayed from a representative blot as an inset. ${ }^{\#} p<0.05$ versus BAPTA/cyclothiazide basal; ${ }^{*} p<0.05$ versus BAPTA/cyclothiazide basal; ${ }^{+} p<0.05$ versus BAPTA/cyclothiazide isoproterenol; $n=4-5$.

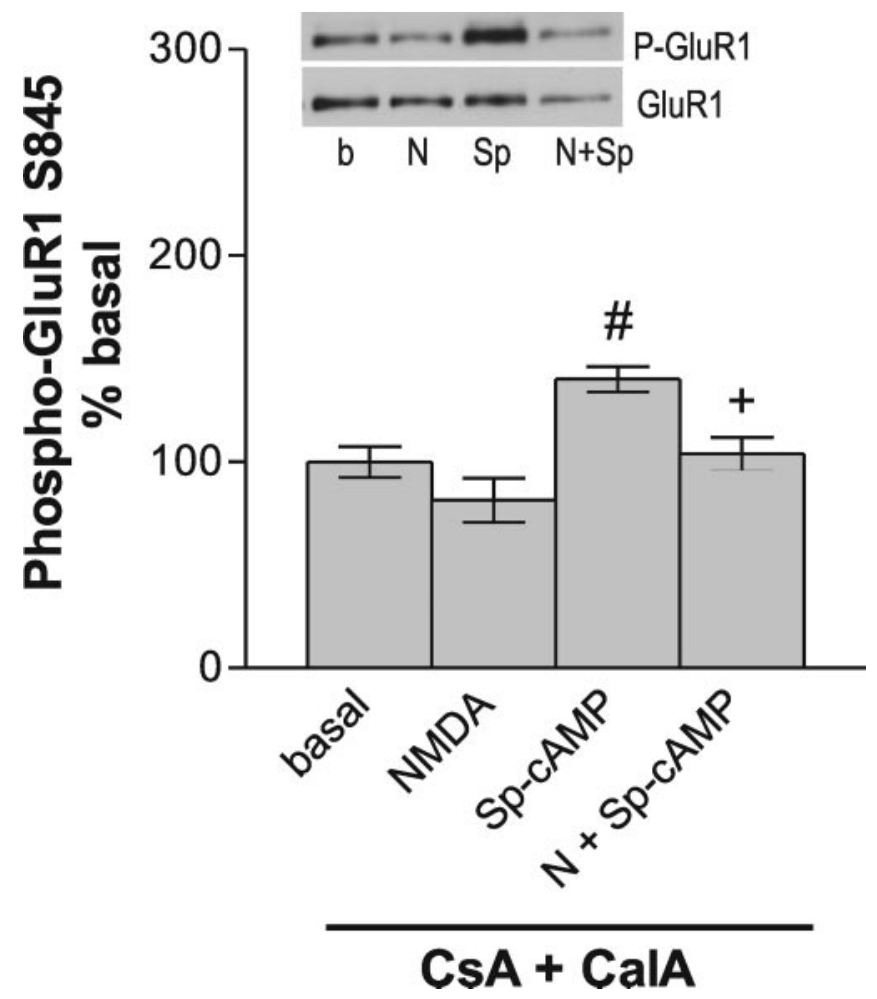

Figure 6. NMDAR activation blocks GluR1 phosphorylation (P-GluR1) downstream of PKA. Hippocampal slices were pretreated for 30 min with $1 \mu \mathrm{m}$ cyclosporine A and $1 \mu \mathrm{m}$ calyculin A (CSA + CalA) before application of $50 \mu \mathrm{m}$ NMDA for $6 \mathrm{~min}(\mathrm{~N}), 25 \mu \mathrm{m}$ Sp-CAMP-AM for $12 \mathrm{~min}$ (Sp), or $25 \mu \mathrm{M}$ Sp-CAMP-AM for 12 min with $50 \mu \mathrm{M}$ NMDA during the final 6 min $(\mathrm{N}+\mathrm{Sp}) . \mathrm{b}$, Basal. Sp-CAMP-AM treatment significantly enhances P-GluR1 at 5845 , and coapplication of NMDA blocks Sp-cAMP-AM-mediated GluR1 phosphorylation at S845. Each treatment condition is displayed from a representative blot as an inset. ${ }^{\#} p<0.05$ versus CsA + CalA basal; ${ }^{+} p<0.05$ versus (sA + CalA Sp-cAMP; $n=7-8$.

GluR1 through the PKA anchoring protein AKAP150 and the scaffolding protein SAP97 (Colledge et al., 2000; Tavalin et al., 2002; Snyder et al., 2005). Although not described as of yet, alterations in protein associations within this complex could serve to prevent PKA access to GluR1. Other reports have demonstrated that glutamatergic activity via both AMPAR and NMDAR regulates trafficking of GluR1containing AMPARs (Carroll et al., 1999; Beattie et al., 2000; Ehlers, 2000; Mangiavacchi and Wolf, 2004), and movement of GluR1 to distinct subcellular compartments could also potentially serve to uncouple PKA from GluR1. However, NMDAR-mediated endocytosis of GluR1 is a $\mathrm{Ca}^{2+}$ - and phosphatase-dependent event. Thus, we predict that the blockade in GluR1 phosphorylation described here is independent of endocytosis. Finally, we must also note the possibility that an atypical $\mathrm{Ca}^{2+}$-independent, depolarizationdependent phosphatase could be recruited that overrides PKA regulation of GluR1 at S845. However, we feel that this is a narrow possibility because of the observation that NMDAR activation in the presence of BAPTA or the phosphatase inhibitors calyculin A and cyclosporin A does not elicit dephosphorylation of GluR1 from the basal state. Thus, activation of an atypical phosphatase that blocks PKA activity must target only a pool of GluR1 phosphorylated downstream of activated PKA and not GluR1 phosphorylated in the basal state.

Although the complete mechanism for blockade of PKAmediated phosphorylation is not yet understood, it is important to recognize this novel means of phospho-protein regulation that is not limited solely to GluR1 but appears to be a mode of regulation for several other PKA substrates as well.

\section{Physiological implications}

The blockade of GluR1 phosphorylation at $\mathrm{S} 845$ described here is likely of physiological significance, because this phenomenon is more readily recruited by NMDAR activation than dephosphorylation of the same site (10 $\mu \mathrm{M}$ NMDA vs $20 \mu \mathrm{M}$ NMDA) (Fig. $2 A$ ). It is interesting to note that Lee et al. (1998) reported depolarization of $\sim 20 \mathrm{mV}$ by application of $10 \mu \mathrm{M}$ NMDA for $10 \mathrm{~min}$ to an acute hippocampal slice preparation, which was insufficient to recruit long-term depression (LTD) or GluR1 dephosphorylation at $\$ 845$. Although our experimental preparations are not directly comparable, these observations suggest that a relatively small level of depolarization is sufficient to recruit blockade of GluR1 phosphorylation by PKA.

Although we have not presented direct evidence that physiologically relevant forms of depolarization recruit the blockade mechanism described here, there is evidence that is consistent with this phenomenon occurring during synaptic plasticity. In previous studies, we and others have reported that NMDAR activation readily recruits robust elevations in cAMP in area CA1 (Chetkovich and Sweatt, 1993; Vanhoose and Winder, 2003), which might be predicted to elicit GluR1 phosphorylation by PKA. However, NMDAR-mediated GluR1 phosphorylation by PKA is not observed, even under conditions that should pro- 
mote PKA coupling to GluR1 (Vanhoose and Winder, 2003). In addition, to date, increases in GluR1 phosphorylation at S845 have not been observed with the induction of LTP (Lee et al., 2000), although phosphorylation of this GluR1 site appears critical for synaptic delivery of GluR1 and LTP (Esteban et al., 2003). These initially perplexing results, which suggest that the NMDAR does not couple to GluR1 phosphorylation at S845 although PKA activity is highly recruited, may now be more readily explained in light of the finding reported here that NMDAR-mediated depolarization blocks PKA regulation of GluR1 at S845. Thus, during LTP induction that recruits NMDAR-mediated PKA activation, GluR1 phosphorylation by PKA is not observed, likely attributable to the simultaneous recruitment of a blockade signal that prevents PKA coupling to GluR1 at $\mathrm{S} 845$.

Although GluR1 phosphorylation at S845 has not yet been reported during LTP induction from a naive state, PKAmediated phosphorylation of this GluR1 site does occur with tetanus-induced, NMDAR-dependent de-depression (Lee et al., 2000), which is LTP induction just after LTD induction. With the induction of LTD, NMDARmediated activity is proposed to elicit dephosphorylation of GluR1 at S845 that is associated with endocytosis of the receptor (Kameyama et al., 1998; Lee et al., 1998; Ehlers, 2000). The observation that NMDAR-mediated dephosphorylation is often transient (Ehlers, 2000; Vanhoose and Winder, 2003) suggests that PKA-mediated phosphorylation of GluR1 can occur subsequent to NMDAR activation. Thus, NMDAR-mediated GluR1 phosphorylation at S845 by PKA may occur, but only when previous GluR1 dephosphorylation of S845 that is associated with endocytosis has been recruited. We speculate that, because NMDAR-mediated dephosphorylation of GluR1 at S845 and LTD are associated with endocytosis of GluR1, it could be that this newly generated pool of endocytic GluR1 is differentially regulated and may not receive regulation via depolarizationmediated blockade of PKA coupling. As with most regulatory mechanisms, there are likely certain conditions under which this mechanism plays a role and other conditions under which this mechanism is not used.

The observation that $\mathrm{G}_{\mathrm{s}}$-coupled receptors readily couple to GluR1 phosphorylation at S845 whereas NMDARs do not leads us to ask the following: what is the benefit for specificity of PKAmediated GluR1 phosphorylation by $\mathrm{G}_{\mathrm{s}}$-coupled receptors relative to NMDARs? Activation of the $\beta$ AR can facilitate NMDARdependent LTP (Thomas et al., 1996; Winder et al., 1999; Gelinas and Nguyen, 2005), and the observation that $\beta A R$ activation recruits and depolarization blocks GluR1 phosphorylation at S845 suggests that this substrate may be a specific target of heterosynaptic input that regulates glutamatergic synaptic plasticity. Because S845 of GluR1 is a critical site that appears to be necessary, but not sufficient, for trafficking of the receptor to the synapse (Esteban et al., 2003), previous heterosynaptic signaling may be a critical signal that sets the stage for the number of GluR1containing AMPARs that are phosphorylated or "primed" to par-
C.

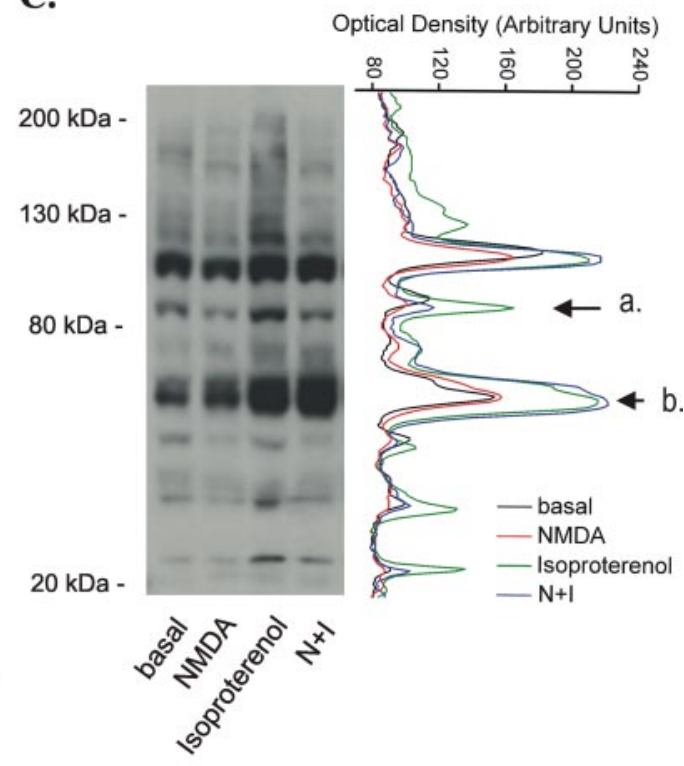

b.

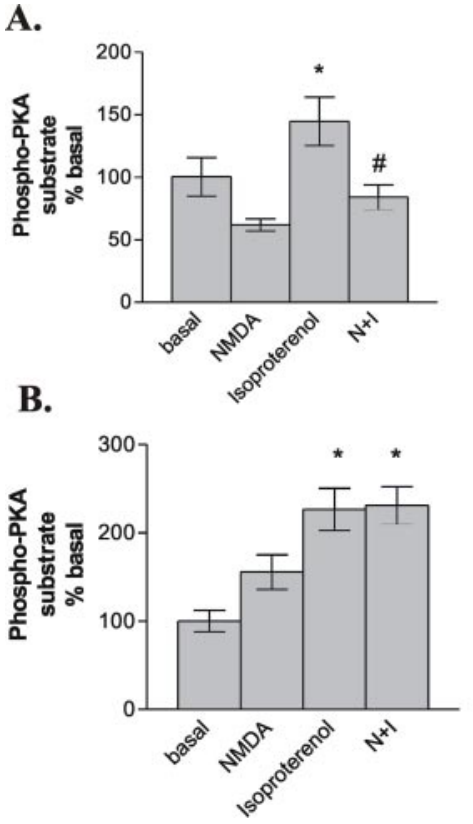

Figure 7. NMDAR activation blocks the phosphorylation of several PKA substrates. Hippocampal slices were treated with 50 $\mu \mathrm{m}$ NMDA for $6 \mathrm{~min}, 1 \mu \mathrm{m}$ isoproterenol for $3 \mathrm{~min}$, and $50 \mu \mathrm{m}$ NMDA for 6 min with $1 \mu \mathrm{m}$ isoproterenol during the final 3 min $(\mathrm{N}+\mathrm{I})$. A, Differences in PKA substrate phosphorylation levels of band "a," indicated on the representative blot, obtained via indicated on the representative blot. $\boldsymbol{C}$, Representative blot with corresponding plot of line scans. ${ }^{*} p<0.05$ versus basal; ${ }^{\#} p<$

ticipate in synaptic plasticity. Importantly, NMDAR-mediated blockade of GluR1 phosphorylation at S845 could be a critical limiting signal that prevents NMDAR activity from targeting all AMPARs to synapses inappropriately. Indeed, one possibility is that activation of neuromodulatory inputs, such as the noradrenergic system, "pre-tags" AMPARs for participation in LTP during subsequent NMDAR activation.

We have identified a novel form of phospho-protein regulation that impacts the phosphorylation state of a critical synaptic substrate, the GluR1 subunit of the AMPAR. In future studies, it will be important to both further delineate mechanisms underlying this blockade phenomenon and more precisely determine its physiological relevance.

\section{References}

Banke TG, Bowie D, Lee H, Huganir RL, Schousboe A, Traynelis SF (2000) Control of GluR1 AMPA receptor function by cAMP-dependent protein kinase. J Neurosci 20:89-102.

Barria A, Derkach V, Soderling T (1997) Identification of the $\mathrm{Ca}^{2+}$ / calmodulin-dependent protein kinase II regulatory phosphorylation site in the alpha-amino-3-hydroxyl-5-methyl-4-isoxazole-propionate-type glutamate receptor. J Biol Chem 272:32727-32730.

Beattie EC, Carroll RC, Yu X, Morishita W, Yasuda H, von Zastrow M, Malenka RC (2000) Regulation of AMPA receptor endocytosis by a signaling mechanism shared with LTD. Nat Neurosci 3:1291-1300.

Ben-Chaim Y, Tour O, Dascal N, Parnas I, Parnas H (2003) The M2 muscarinic G-protein-coupled receptor is voltage-sensitive. J Biol Chem 278:22482-22491.

Carroll RC, Beattie EC, Xia H, Luscher C, Altschuler Y, Nicoll RA, Malenka RC, von Zastrow M (1999) Dynamin-dependent endocytosis of ionotropic glutamate receptors. Proc Natl Acad Sci USA 96:14112-14117.

Chao SZ, Lu W, Lee HK, Huganir RL, Wolf ME (2002) D(1) dopamine receptor stimulation increases GluR1 phosphorylation in postnatal nucleus accumbens cultures. J Neurochem 81:984-992.

Chetkovich DM, Sweatt JD (1993) NMDA receptor activation increases cyclic AMP in area CA1 of the hippocampus via calcium/calmodulin stimulation of adenylyl cyclase. J Neurochem 61:1933-1942. 
Colledge M, Dean RA, Scott GK, Langeberg LK, Huganir RL, Scott JD (2000) Targeting of PKA to glutamate receptors through a MAGUK-AKAP complex. Neuron 27:107-119.

Cull-Candy SG, Leszkiewicz DN (2004) Role of distinct NMDA receptor subtypes at central synapses. Sci STKE 2004: re16.

Derkach VA (2003) Silence analysis of AMPA receptor mutated at the CaMkinase II phosphorylation site. Biophys J 84:1701-1708.

Derkach V, Barria A, Soderling TR (1999) $\mathrm{Ca}^{2+} /$ calmodulin-kinase II enhances channel conductance of alpha-amino-3-hydroxy-5-methyl-4isoxazolepropionate type glutamate receptors. Proc Natl Acad Sci USA 96:3269-3274.

Ehlers MD (2000) Reinsertion or degradation of AMPA receptors determined by activity-dependent endocytic sorting. Neuron 28:511-525.

Esteban JA, Shi SH, Wilson C, Nuriya M, Huganir RL, Malinow R (2003) PKA phosphorylation of AMPA receptor subunits controls synaptic trafficking underlying plasticity. Nat Neurosci 6:136-143.

Gelinas JN, Nguyen PV (2005) $\beta$-Adrenergic receptor activation facilitates induction of a protein synthesis-dependent late phase of long-term potentiation. J Neurosci 25:3294-3303.

Hatton CJ, Paoletti P (2005) Modulation of triheteromeric NMDA receptors by N-terminal domain ligands. Neuron 46:261-274.

Kameyama K, Lee HK, Bear MF, Huganir RL (1998) Involvement of a postsynaptic protein kinase A substrate in the expression of homosynaptic long-term depression. Neuron 21:1163-1175.

Lee HK, Kameyama K, Huganir RL, Bear MF (1998) NMDA induces longterm synaptic depression and dephosphorylation of the GluR1 subunit of AMPA receptors in hippocampus. Neuron 21:1151-1162.

Lee HK, Barbarosie M, Kameyama K, Bear MF, Huganir RL (2000) Regulation of distinct AMPA receptor phosphorylation sites during bidirectional synaptic plasticity. Nature 405:955-959.

Lee HK, Takamiya K, Han JS, Man H, Kim CH, Rumbaugh G, Yu S, Ding L, He C, Petralia RS, Wenthold RJ, Gallagher M, Huganir RL (2003) Phosphorylation of the AMPA receptor GluR1 subunit is required for synaptic plasticity and retention of spatial memory. Cell 112:631-643.

Linden DJ, Smeyne M, Connor JA (1993) Induction of cerebellar long-term depression in culture requires postsynaptic action of sodium ions. Neuron 11:1093-1100.

Maas Jr JW, Vogt SK, Chan GC, Pineda VV, Storm DR, Muglia LJ (2005) Calcium-stimulated adenylyl cyclases are critical modulators of neuronal ethanol sensitivity. J Neurosci 25:4118-4126.

Mammen AL, Kameyama K, Roche KW, Huganir RL (1997) Phosphorylation of the alpha-amino-3-hydroxy-5-methylisoxazole4-propionic acid receptor GluR1 subunit by calcium/calmodulin-dependent kinase II. J Biol Chem 272:32528-32533.
Mangiavacchi S, Wolf ME (2004) Stimulation of $N$-methyl-D-aspartate receptors, AMPA receptors or metabotropic glutamate receptors leads to rapid internalization of AMPA receptors in cultured nucleus accumbens neurons. Eur J Neurosci 20:649-657.

Price CJ, Kim P, Raymond LA (1999) D1 dopamine receptor-induced cyclic AMP-dependent protein kinase phosphorylation and potentiation of striatal glutamate receptors. J Neurochem 73:2441-2446.

Roche KW, O’Brien RJ, Mammen AL, Bernhardt J, Huganir RL (1996) Characterization of multiple phosphorylation sites on the AMPA receptor GluR1 subunit. Neuron 16:1179-1188.

Snyder EM, Colledge M, Crozier RA, Chen WS, Scott JD, Bear MF (2005) Role for A kinase-anchoring proteins (AKAPS) in glutamate receptor trafficking and long term synaptic depression. J Biol Chem 280:16962-16968.

Snyder GL, Allen PB, Fienberg AA, Valle CG, Huganir RL, Nairn AC, Greengard P (2000) Regulation of phosphorylation of the GluR1 AMPA receptor in the neostriatum by dopamine and psychostimulants in vivo. J Neurosci 20:4480-4488.

Snyder GL, Galdi S, Fienberg AA, Allen P, Nairn AC, Greengard P (2003) Regulation of AMPA receptor dephosphorylation by glutamate receptor agonists. Neuropharmacology 45:703-713.

Tavalin SJ, Colledge M, Hell JW, Langeberg LK, Huganir RL, Scott JD (2002) Regulation of GluR1 by the A-kinase anchoring protein 79 (AKAP79) signaling complex shares properties with long-term depression. J Neurosci 22:3044-3051.

Thomas MJ, Moody TD, Makhinson M, O’Dell TJ (1996) Activitydependent beta-adrenergic modulation of low frequency stimulation induced LTP in the hippocampal CA1 region. Neuron 17:475-482.

Vanhoose AM, Winder DG (2003) NMDA and $\beta_{1}$-adrenergic receptors differentially signal phosphorylation of glutamate receptor type 1 in area CA1 of hippocampus. J Neurosci 23:5827-5834.

Vinade L, Dosemeci A (2000) Regulation of the phosphorylation state of the AMPA receptor GluR1 subunit in the postsynaptic density. Cell Mol Neurobiol 20:451-463.

Winder DG, Martin KC, Muzzio IA, Rohrer D, Chruscinski A, Kobilka B, Kandel ER (1999) ERK plays a regulatory role in induction of LTP by theta frequency stimulation and its modulation by beta-adrenergic receptors. Neuron 24:715-726.

Yu XM, Salter MW (1998) Gain control of NMDA-receptor currents by intracellular sodium. Nature 396:469-474.

Zhang J, Ma Y, Taylor SS, Tsien RY (2001) Genetically encoded reporters of protein kinase A activity reveal impact of substrate tethering. Proc Natl Acad Sci USA 98:14997-15002. 